\title{
On the Derivation of a Closed-Form Expression for the Solutions of a Subclass of Generalized Abel Differential Equations
}

\author{
Panayotis E. Nastou, ${ }^{1}$ Paul Spirakis, ${ }^{2,3}$ Yannis C. Stamatiou, ${ }^{3,4}$ and Apostolos Tsiakalos ${ }^{5}$ \\ ${ }^{1}$ Department of Mathematics, University of Aegean, 83200 Samos, Greece \\ ${ }^{2}$ Department of Computer Engineering and Informatics, University of Patras Rio, 26504 Patras, Greece \\ ${ }^{3}$ Computer Technology Institute and Press "Diophantus" Rio, 26504 Patras, Greece \\ ${ }^{4}$ Department of Business Administration, University of Patras Rio, 26504 Patras, Greece \\ ${ }^{5}$ Department of Mathematics, University of Ioannina, 45110 Ioannina, Greece
}

Correspondence should be addressed to Panayotis E. Nastou; pnastou@aegean.gr

Received 9 March 2013; Accepted 30 May 2013

Academic Editor: Elena Braverman

Copyright (C) 2013 Panayotis E. Nastou et al. This is an open access article distributed under the Creative Commons Attribution License, which permits unrestricted use, distribution, and reproduction in any medium, provided the original work is properly cited.

We investigate the properties of a general class of differential equations described by $d y(t) / d t=f_{k+1}(t) y(t)^{k+1}+f_{k}(t) y(t)^{k}+\cdots+$ $f_{2}(t) y(t)^{2}+f_{1}(t) y(t)+f_{0}(t)$, with $k>1$ a positive integer and $f_{i}(t), 0 \leq i \leq k+1$, with $f_{i}(t)$, real functions of $t$. For $k=2$, these equations reduce to the class of Abel differential equations of the first kind, for which a standard solution procedure is available. However, for $k>2$ no general solution methodology exists, to the best of our knowledge, that can lead to their solution. We develop a general solution methodology that for odd values of $k$ connects the closed form solution of the differential equations with the existence of closed-form expressions for the roots of the polynomial that appears on the right-hand side of the differential equation. Moreover, the closed-form expression (when it exists) for the polynomial roots enables the expression of the solution of the differential equation in closed form, based on the class of Hyper-Lambert functions. However, for certain even values of $k$, we prove that such closed form does not exist in general, and consequently there is no closed-form expression for the solution of the differential equation through this methodology.

\section{Introduction}

In [1], a differential equation was derived, in the context of the theoretical analysis of a security protocol, that can be written in the following form:

$$
\begin{aligned}
(1-t) & \frac{d y(t)}{d t} \\
= & \left(\begin{array}{c}
k-1 \\
{\left[\frac{k}{2}\right]-1}
\end{array}\right) y(t)^{k-\lceil k / 2\rceil+1}(1-y(t))^{\lceil k / 2\rceil} .
\end{aligned}
$$

In [2], the differential equations (1) are written as partial fractions in such a way that the form of their solution matches the recursive pattern of the definition of the Hyper-Lambert functions that were proposed in [3] as a generalization of the well-known Lambert $W$ function (see [4] for an introduction to this function and its properties). The differential equations defined by (1) can be seen as belonging to the general differential equation form

$$
\begin{aligned}
\frac{d y(t)}{d t}= & f_{k+1}(t) y(t)^{k+1}+f_{k}(t) y(t)^{k} \\
& +\cdots+f_{2}(t) y(t)^{2}+f_{1}(t) y(t)+f_{0}(t),
\end{aligned}
$$

with $f_{i}(t), 0 \leq i \leq k+1$ real functions. The class defined by (2) appears to generalize, naturally, the Abel class of differential equations (see [5]). Unfortunately, no general solution strategy exists (to the best of our knowledge) for the solution of this general class for $k>2$, in analogy with the methodology that exists for the Abel class. Thus, we could not reduce the problem of finding the solution of (1) into the problem of solving (2) for various values of $k$. 
In this paper, we propose a general methodology which for odd values of $k>2$ provides a closed-form expression of the solution of (1) based on closed-form solutions of the roots of the equation $g(y(t))-s$ where $g(y(t))=$ $\left(\begin{array}{c}k-1 \\ \lceil k / 2\rceil-1\end{array}\right) y(t)^{k-\lceil k / 2\rceil+1}(1-y(t))^{\lceil k / 2\rceil}$ (the polynomial on the right-hand side of (1)) and $s \neq 0$. Moreover, this closed-form solutions are linked with the generalized Hyper-Lambert functions.

However, for certain even values of $k>2$ (at least for values of $k$ where $k+1$ is prime), we prove that the roots of $g(y(t))-s$ cannot be expressed by radicals, and, thus, no closed-form solution can be obtained using this methodology. This latter result may be of independent interest.

\section{Properties of the Target Class of Differential Equations}

The target differential equation class is the following (see [1] for its derivation):

$$
\begin{aligned}
\frac{d x(t)}{d t}= & -\sum_{j=\lceil k / 2\rceil}^{k}\left(\begin{array}{c}
k \\
j
\end{array}\right)\left(1-\frac{x(t)}{(1-t)}\right)^{j}\left(\frac{x(t)}{1-t}\right)^{k-j} \\
& \times\left(1-\frac{j}{k}\right)+\left[-\frac{x(t)}{(1-t)}\right] \\
& \times \sum_{j=0}^{\lceil k / 2\rceil-1}\left(\begin{array}{l}
k \\
j
\end{array}\right) \times\left(1-\frac{x(t)}{(1-t)}\right)^{j}\left(\frac{x(t)}{1-t}\right)^{k-j} .
\end{aligned}
$$

If we set $y(t)=x(t) /(1-t)$, (3) becomes

$$
\begin{aligned}
\frac{d y(t)}{d t}= & \frac{y(t)}{1-t} \\
& \times\left(1-\sum_{j=0}^{\lceil k / 2\rceil-1}\left(\begin{array}{c}
k \\
j
\end{array}\right)(1-y(t))^{j} y(t)^{k-j}\right)-\frac{1}{1-t} \\
& \times \sum_{j=\lceil k / 2\rceil}^{k}\left(\begin{array}{c}
k \\
j
\end{array}\right)\left(1-\frac{j}{k}\right)(1-y(t))^{j} y(t)^{k-j} .
\end{aligned}
$$

After some algebraic manipulations, (4) becomes

$$
\begin{aligned}
(1-t) & \frac{d y(t)}{d t} \\
= & \sum_{j=\lceil k / 2\rceil}^{k}\left(\begin{array}{l}
k \\
j
\end{array}\right)(1-y(t))^{j} y(t)^{k-j}\left(1-\frac{j}{k}-y\right) .
\end{aligned}
$$

Let $g(y(t))$ be the polynomial, in $y(t)$, at the right-hand side of the differential equation (5). Throughout this paper, when we refer to this polynomial, we will always mean that the polynomial is in $y(t)$, treating $y(t)$ as an independent variable. Based on the following two lemmas, whose proofs follow by straightforward algebraic manipulations, we will present the properties of the class of ordinary differential equations defined by (5).
Lemma 1. For any $l, 0 \leq l \leq k$, and independently of $y$, it holds

$$
\sum_{j=l}^{k}\left(\begin{array}{c}
k \\
j
\end{array}\right) y^{l-1-j}(1-y)^{j-l}\left[\left(1-\frac{j}{k}\right)-y\right]=-\left(\begin{array}{c}
k-1 \\
l-1
\end{array}\right) .
$$

Based on Lemma 1, the following can be proved.

Lemma 2. The right-hand side of the differential equation (5) can be written as follows:

$$
g(y(t))=\left(\begin{array}{c}
k-1 \\
\left\lceil\frac{k}{2}\right\rceil-1
\end{array}\right) y(t)^{k-\lceil k / 2\rceil+1}(1-y(t))^{\lceil k / 2\rceil} .
$$

From Lemma 2, the following property of the polynomial $g(y(t))$ can be readily derived.

Corollary 3. The roots of the polynomial $g(y(t))$ are 0 with multiplicity $\lfloor k / 2\rfloor+1$ and 1 with multiplicity $\lceil k / 2\rceil$.

Lemma 4. The solution $y(t)$ of the differential equation (5) with $y(0) \in[0,1)$ is a function that has the following two properties: (i) all its values are real numbers in the interval $(0,1)$, and (ii) it is monotonically increasing.

Proof. Since $y(0) \in[0,1)$, because $0 \leq x(0)<1$ and $t=$ 0 , and the polynomial $g(y(t))$ contains the factors $y(t)$ and $(1-y(t))$ and its roots are 0 and 1 , the solution function $y(t)$ of the differential equation (5) cannot get values below 0 or above 1 . Thus, statement (i) holds.

Since $d y(t) / d t$ is always positive, as $g(y(t))$ is always positive for $y(t) \in(0,1)$ and $1-t>0$, then $y(t)$ is a monotonically increasing function, thus proving statement (ii).

Lemma 5. The roots of the first derivative of $g(y(t))$ as a function of $y(t)$ are $w_{1}=0$ with multiplicity $\lfloor k / 2\rfloor, w_{2}=1$ with multiplicity $\lceil k / 2\rceil$, and $w_{3}=(k+1-\lceil k / 2\rceil) /(k+1)$, while $w_{3}$ is a maximum point of $g(y(t))$.

Proof. Since $g(y(t))$ is continuous on $[0,1], g(0)=g(1)=0$, and it is differentiable on $(0,1)$, there is $w \in(0,1)$ where $d g(y(t)) /\left.d y(t)\right|_{y(t)=w}=0$ and $g(y(t))$ attains minimum and maximum values on $[0,1]$. After some algebraic manipulations we obtain a closed formula for the first derivative of $g(y(t))$ :

$$
\begin{aligned}
& \frac{d g(y(t))}{d y(t)}=\left(\begin{array}{c}
k-1 \\
\left\lceil\frac{k}{2}\right\rceil-1
\end{array}\right)\left(k+1-\left\lceil\frac{k}{2}\right\rceil \frac{1}{1-y(t)}\right) \\
& \times y(t)^{k-\lceil k / 2\rceil}(1-y(t))^{\lceil k / 2\rceil} \text {. }
\end{aligned}
$$

From (8), it is readily derived that the roots of the first derivative of $g(y(t))$ are $w_{1}=0$ with multiplicity $\lfloor k / 2\rfloor$, $w_{2}=1$ with multiplicity $\lceil k / 2\rceil$, and $w_{3}=(k+1-\lceil k / 2\rceil) /(k+1)$. 
The second derivative of $g(y(t))$ is given by the following formula:

$$
\begin{aligned}
\frac{1}{\left(\begin{array}{c}
k-1 \\
\lceil k / 2\rceil-1
\end{array}\right)} \frac{d^{2} g(y(t))}{d y(t)} \\
=\left(k-\left\lceil\frac{k}{2}\right\rceil\right) w(t) y(t)^{k-\lceil k / 2\rceil-1} \\
\quad \times(1-y(t))^{\lceil k / 2\rceil}-\left\lceil\frac{k}{2}\right\rceil w(t) y(t)^{k-\lceil k / 2\rceil} \\
\quad \times(1-y(t))^{\lceil k / 2\rceil-1}-\left\lceil\frac{k}{2}\right\rceil y(t)^{k-\lceil k / 2\rceil} \\
\quad \times(1-y(t))^{\lceil k / 2\rceil-2},
\end{aligned}
$$

where $w(t)=k+1-\lceil k / 2\rceil(1 /(1-y(t)))$. For $y(t)=w_{3}$, the factor $w(t)$ of (9) becomes 0 , and thus a negative term remains. Thus $d^{2} g(y(t)) / d y(t)<0$ on $[0,1]$ which means that $w_{3}$ is a maximum point of $g(y(t))$.

Lemma 6. The function $g(y(t))$ as function of $y(t)$ is not monotonic on $[0,1]$, and it is an increasing function on $\left(0, w_{3}\right)$ and a decreasing function on $\left(w_{3}, 1\right)$.

Proof. Since $y(t) \in(0,1)$, the sign of the first derivative of $g(y(t))$ in $(8)$ is determined by the factor $(k+1-\lceil k / 2\rceil(1 /(1-$ $y(t))))$. Specifically, it holds that $(d g(y(t)) / d y(t))>0$ for $y(t) \in\left(0, w_{3}\right)$ and $(d g(y(t)) / d y(t))<0$ for $y(t) \in\left(w_{3}, 1\right)$. Since the $d g(y(t)) / d y(t)$ changes sign on $[0,1]$, the function $g(y(t))$ is not monotonic, but it is an increasing function on $\left(0, w_{3}\right)$ and a decreasing function on $\left(w_{3}, 1\right)$.

Lemma 7. For odd $k, w_{3}=1 / 2$ and $g\left(w_{3}\right)=\left(\begin{array}{c}k-1 \\ \lceil k / 2\rceil-1\end{array}\right)$ $(1 / 2)^{2\lceil k / 2\rceil}$, while for even $k, w_{3}=1-(k /(2(k+1)))>1 / 2$ and $\lim _{k \rightarrow \infty} w_{3}=1 / 2$.

Proof. For an odd $k$, it holds $\lceil k / 2\rceil=(k+1) / 2$ and $k-\lceil k / 2\rceil+$ $1=\lceil k / 2\rceil$. Thus,

$$
w_{3}=\frac{k+1-\lceil k / 2\rceil}{k+1}=\frac{1}{2},
$$

and by applying $y(t)=w_{3}$ in (7), we obtain the following:

$$
g\left(w_{3}\right)=\left(\begin{array}{c}
k-1 \\
\left\lceil\frac{k}{2}\right\rceil-1
\end{array}\right)\left(\frac{1}{2}\right)^{2\lceil k / 2\rceil} .
$$

For even $k$, it holds $\lceil k / 2\rceil=k / 2$. Consequently, we obtain

$$
w_{3}=1-\frac{k}{2(k+1)}
$$

which means that $w_{3}>1 / 2$. Applying L'Hospital's rule, for large even $k$, we have that $\lim _{k \rightarrow \infty} w_{3}=1 / 2$.

The following theorem gives the specific form of the polynomial function $g(y(t))$ of (5).
TABLE 1: The polynomial of the right-hand side of (13) for various values of $k$.

\begin{tabular}{lc}
\hline$k$ & $g(y(t))$ \\
\hline 2 & $-y(t)^{3}+y(t)^{2}$ \\
3 & $2 y(t)^{4}-4 y(t)^{3}+2 y(t)^{2}$ \\
4 & $3 y(t)^{5}-6 y(t)^{4}+3 y(t)^{3}$ \\
5 & $-6 y(t)^{6}+18 y(t)^{5}-18 y(t)^{4}+6 y(t)^{3}$ \\
6 & $-10 y(t)^{7}+30 y(t)^{6}-30 y(t)^{5}+10 y(t)^{4}$ \\
7 & $20 y(t)^{8}-80 y(t)^{7}+120 y(t)^{6}-80 y(t)^{5}+20 y(t)^{4}$ \\
8 & $35 y(t)^{9}-140 y(t)^{8}+210 y(t)^{7}-140 y(t)^{6}+35 y(t)^{5}$ \\
\hline
\end{tabular}

Theorem 8. The right-hand side $g(y(t))$ of differential equation (5) is a polynomial function of one argument of $(k+1)$ degree, and the minimum degree of its terms is $k-\lceil k / 2\rceil+1$; that is, it has the form:

$$
(1-t) \frac{d y(t)}{d t}=g(y(t))=\sum_{j=0}^{\lceil k / 2\rceil} a_{j} \cdot y(t)^{s+j},
$$

where $s=k-\lceil k / 2\rceil+1$ and

$$
a_{j}=(-1)^{j}\left(\left\lceil\begin{array}{c}
\frac{k}{2} \\
j
\end{array}\right]\right)\left(\begin{array}{c}
k-1 \\
\left\lceil\frac{k}{2}\right\rceil-1
\end{array}\right) .
$$

Proof. Based on (7) and the binomial theorem, the results of the theorem can be readily deduced.

Lemma 9. The function $g$ is concave on $[0,1]$.

Proof. The interval $[0,1]$ is convex, since every interval in $\mathbb{R}^{1}$ is convex. Thus, for $0 \leq \lambda \leq 1$, the convex combination of any $y\left(t_{1}\right)$ and $y\left(t_{2}\right), y\left(t_{3}\right)=\lambda y\left(t_{1}\right)+(1-\lambda) y\left(t_{2}\right)$ is in the interval $[0,1]$. If $\lambda$ is close to 0 , the convex combination is close to $y\left(t_{2}\right)$. Based on Lemma 6 , it is obvious that $g\left(y\left(t_{3}\right)\right) \geq$ $\lambda g\left(y\left(t_{1}\right)\right)+(1-\lambda) g\left(y\left(t_{2}\right)\right)$, which means that the function $g$ is concave.

Table 1 presents the polynomial function of the righthand side of the differential equation using Theorem 8 for various values of $k$.

Some plots of the polynomial function $g(y(t))$ as a function of $y(t)$ in the same graph for various odd values of $k$ appear in Figure 1. It is easy to verify the result of Lemma 7 that the local maxima of $g$ appear at $y(t)=0.5$ independently of $k$ value. Moreover, the function $g$ is symmetric around the axis $y(t)=0.5$; that is, for every value $g(y(t)) \leq g(0.5)$, there are $y\left(t_{1}\right)<0.5, y\left(t_{2}\right)>0.5$, and $\left|y\left(t_{1}\right)-0.5\right|=$ $\left|y\left(t_{2}\right)-0.5\right|$ where $g\left(y\left(t_{1}\right)\right)=g\left(y\left(t_{2}\right)\right)$. By solving the differential equation (5) for a certain odd value of $k$ where $g(y(t)) \in(0, g(0.5))$, we can calculate $t$ that $g(y(t))$ gets its maximum value; that is, the first derivative of $y(t)$ becomes maximum. This value of $t$ along with the fact that $g(y(t))$ is symmetric around the axis $y(t)=0.5$ can be used to calculate the time value at which the sought function attains a specific target value, which can be of use in determining a particular stopping time for the protocol given in [1]. 


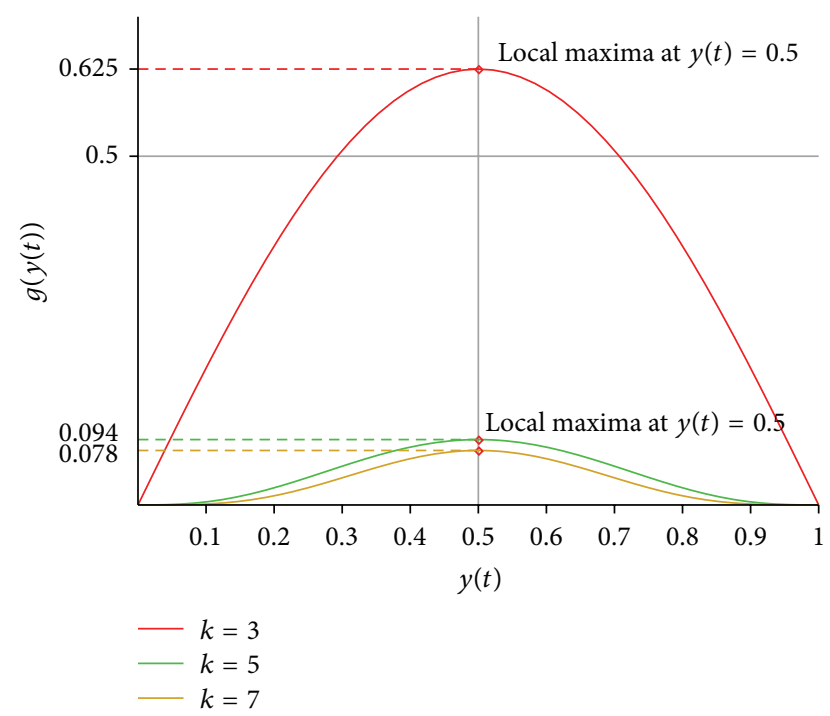

FIGURE 1: A plot of $g(y(t))$ as a function of $y(t)$ for odd values of $k$.

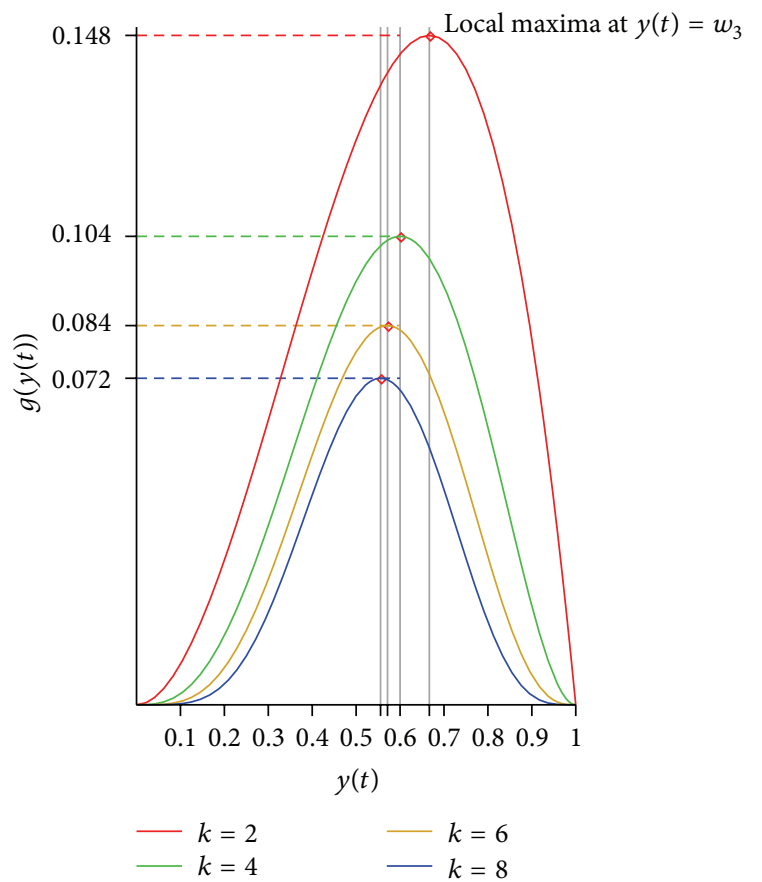

Figure 2: A plot of $g(y(t))$ as a function of $y(t)$ for even values of $k$.

Figure 2 presents some plots of $g$ as a function of $y(t)$ in the same graph for various even values of $k$. In these plots, the local maxima of $g$ appear at $y(t)=0.66,0.6,0.57$, and 0.55 for $k=2,4,6,8$ correspondingly. Moreover, as $k$ increases, the local maxima of $g$ are obtained at a $y(t)$ closer to 0.5 . But in practice, $k$ takes small values. Moreover, the range $\left(0, g\left(w_{3}\right)\right)$ where the function $g$ is an increasing function is longer than the range $\left(g\left(w_{3}\right), 1\right)$ where the function $g$ is a decreasing function; that is, the values of $g$ are not equally balanced around axis $y(t)=w_{3}$.
Theorem 10. For $s \in\left(0, g\left(w_{3}\right)\right)$, the equation $g(y(t))=s$ has always one real root $r_{1}(s)$ within the interval $\left(0, w_{3}\right)$ and one real root $r_{2}(s)$ within the interval $\left(w_{3}, 1\right)$. In addition, the root $r_{1}(s)$ is a monotonically increasing function of $s$, while $r_{2}(s)$ is a monotonically decreasing function of $s$.

Proof. Let $p(y(t))=g(y(t))-s$. Since the first derivative of $p(y(t))$ is equal to the first derivative of $g(y(t))$, from Lemma 5, it follows that $w_{3}$ is also a root of $d p(y(t)) / d t$. By Lemmas 5 and 6 we conclude that the polynomial $p(y(t))$ attains its maximum at $w_{3}$. Moreover, it is monotone increasing for $y(t)<w_{3}$ and monotone decreasing for $y(t)>$ $w_{3}$.

It holds $p(0)=p(1)=-s<0$, while $\left.p\left(w_{3}\right)=g\left(w_{3}\right)-s\right\rangle$ 0 since $s \in\left(0, g\left(w_{3}\right)\right)$. Thus, since $p(y(t))$ as a function of $y(t)$ is continuous on $(0,1)$ and it has opposite signs at the endpoints of intervals $\left(0, w_{3}\right)$ and $\left(w_{3}, 1\right)$, it has one real solution $r_{1}(s)$ in the first interval and one real solution $r_{2}(s)$ in the second one.

Finally, due to the monotonicity of $p(y(t))$ in each of these intervals, the root $r_{1}(s)$ monotonically increases as $s$ increases, while the root $r_{2}(s)$ monotonically decreases.

Lemma 11. For odd $k$, it holds $r_{1}(s)+r_{2}(s)=1$.

Proof. From Lemma 7, $w_{3}=1 / 2$. Thus $r_{1}(s) \in(0,1 / 2)$ and $r_{2}(s) \in(1 / 2,1)$. Since $p(y(t))$ as a function of $y(t)$ is continuous on $(0,1)$ and it has opposite signs at the endpoints of intervals $(0,1 / 2)$ and $(1 / 2,1)$, the roots $r_{1}(s)$ and $r_{2}(s)$ can be approximated by the use of the bisection method.

After $n$ iterations of the bisection method on these intervals, the calculated roots $r_{1}(s)$ and $r_{2}(s)$ satisfy the identity $r_{1}(s)+r_{2}(s)=1$ since the intervals are balanced, $g\left(r_{1}(s)\right)=g\left(r_{2}(s)\right)=s$, and in each iteration the method operates in one of the two halves of the two intervals.

\section{The Solution of the Class of the Differential Equations for Odd $k$}

By the class of differential equations defined by (13), it is easy to see that it has the form $d y(t) / d t=\sum_{i=0}^{k+1} f_{i}(t) y(t)^{i}$, with $k>$ 1 and $f_{i}(t), 0 \leq i \leq k+1$, specific real functions of $t$. This form appears to generalize the class of Abel differential equations of the first kind in that it involves the sought function to powers greater than 3 , for $k \geq 3$. To the best of our knowledge, no general solution strategy exists for generalized differential equations of the Abel type that can be exploited to solve (13). In this section we will describe such a solution strategy for odd $k$ that makes use of the result of Lemma 4 and the properties of the polynomial function $g(y(t))$ that have been proved in Section 2.

Definition 12. Let $I$ be an index set, and let $f_{i}: \mathbb{C} \rightarrow \mathbb{C}$ be arbitrary complex functions which do not vanish identically. Assuming $m, n \in \mathbb{N}$ such that $m \geq n$, we define $F_{n, m}(z)$ : $\mathbb{N}^{2} \times \mathbb{C} \rightarrow \mathbb{C}$ as follows:

$$
F_{n, m}(z)= \begin{cases}e^{z} & \text { if } n=1 \\ e^{f_{m-(n-1)}(z) F_{n-1, m}(z)} & \text { if } n>1 .\end{cases}
$$


Definition 13. Let $f_{i}$ be as in Definition 12 and $z \in \mathbb{C}$. Then we define

$$
G\left(f_{1}(z), f_{2}(z), \ldots, f_{j}(z) ; z\right)=z F_{j+1, j+1}(z) .
$$

Definition 14. Let $f_{i}$ be as in Definition 12, $G$ as in Definition 13, and $y \in \mathbb{C}$. Then the function $\operatorname{HW}\left(\left\{f_{i}\right\}_{i \in I} ; y\right)$ is the function which satisfies the following equation:

$$
G\left(\left\{f_{i}\right\}_{i \in I} ; \operatorname{HW}\left(\left\{f_{i}\right\}_{i \in I} ; y\right)\right)=y .
$$

If we set $s \in \mathbb{R}_{+}$at the right side of (1), we obtain the following functional equation:

$$
(1-t) \frac{d y(t)}{d t}=s .
$$

Our approach relies on the following fact for the solution $t(s)$ of the functional equation (18).

Claim 1. Let $r(s)$ be a real function in $\mathbb{R}$, such that each value of $t^{-1}$ belongs to the domain of $r(s)$ for every value $s \in \mathbb{R}_{+}$. Assuming that $b=t(s)$, then $y(b)=y(t(s))=r\left(t^{-1}(b)\right)$.

Lemma 15. The solution $t(s), s \in \mathbb{R}_{+}$, of the functional equation

$$
(1-t) \frac{d y(t)}{d t}=s
$$

forms a solution of the equation $g(y(t))=s$.

Proof. Given $s \in \mathbb{R}_{+}$and the solution $t(s)$ to (18), it follows from (13) that $y(t(s))$ is a root of the polynomial equation $g(y(t))=s$.

Theorem 16. For odd $k$ and $s \in \mathbb{R}_{+}$, the solution $t(s)$ of the functional equation (18) is equal to

$$
t(s)=1+c e^{-\int(1 / s)(d r(s) / d s) d s}
$$

with $r(s)=(1 \pm \sqrt{1-4 h(s)}) / 2$, where $h(s)=$ $\left(s /\left(\begin{array}{c}k-1 \\ \lceil k / 2\rceil-1\end{array}\right)\right)^{1 /\lceil k / 2\rceil}$ and the real positive solution of the equation $g(y(t))=s$.

Proof. For odd $k$ the exponents of $y(t)$ and $(1-y(t))$ of the polynomial $g(y(t))$ in $(7)$ become equal, that is, $k-\lceil k / 2\rceil+1=$ $\lceil k / 2\rceil=l$, and the polynomial $g(y(t))$ could be written as follows:

$$
g(y(t))=\left(\begin{array}{c}
k-1 \\
\left\lceil\frac{k}{2}\right\rceil-1
\end{array}\right) y(t)^{l}(1-y(t))^{l} .
$$

Based on (21), the polynomial equation $g(y(t))=s$ becomes

$$
y(t)(1-y(t))=\sqrt[4]{\frac{s}{\left(\begin{array}{c}
k-1 \\
\lceil k / 2\rceil-1
\end{array}\right)} .}
$$

Let $h(s)=\sqrt[l]{s /\left(\begin{array}{c}k-1 \\ \lceil k / 2\rceil-1\end{array}\right)}$, the right-hand side of (22). By Lemmas 5 and 7 , we have $s \leq\left(\begin{array}{c}k-1 \\ \lceil k / 2\rceil-1\end{array}\right)\left(1 /\left(2^{2\lceil k / 2\rceil}\right)\right)$ which leads after some algebraic manipulations to $4 h(s) \leq 1$. This means that the discriminant of the quadratic equation (22) $\Delta=1-4 h(s)$ is positive, and consequently by applying the closed-form formula for the solutions of the quadratic polynomial equation (22), we find that the solutions are the following:

$$
r_{1}(s)=\frac{1+\sqrt{1-4 h(s)}}{2}, \quad r_{2}(s)=\frac{1-\sqrt{1-4 h(s)}}{2} .
$$

It is obvious that $r_{1}(s)+r_{2}(s)=1$. Thus, $r_{1}(s)$ and $r_{2}(s)$ are real valued functions, where $r_{1}(s)$ is a monotonically decreasing function with $w_{3} \leq r_{1}(s) \leq 1$ and $r_{2}(s)$ is a monotonically increasing function with $0 \leq r_{2}(s) \leq w_{3}$. By Theorem 10 and Lemma 11, the roots $r_{1}(s)$ and $r_{2}(s)$ are the appropriate roots, and consequently we set $r(s)=r_{2}(s)$ for $y(t) \in[0,0.5]$ and $r(s)=r_{1}(s)$ for $y(t) \in(0.5,1]$.

Using the chain differentiation rule, we obtain the following:

$$
y(t(s))=r(s) \Longrightarrow\left(\frac{d y(t)}{d t}\right)_{t=t(s)} \frac{d t(s)}{d s}=\frac{d r(s)}{d s} .
$$

However, since $(1-t)(d y(t) / d t)=s$ for $t=t(s)$, it follows that

$$
\left(\frac{d y(t)}{d t}\right)_{t=t(s)}=\frac{s}{1-t(s)} .
$$

Thus, (24) becomes

$$
\left(\frac{d y(t)}{d t}\right)_{t=t(s)} \frac{d t(s)}{d s}=\frac{d r(s)}{d s} \Longrightarrow \frac{s}{1-t(s)} \frac{d t(s)}{d s}=\frac{d r(s)}{d s} .
$$

Solving the differential equation in (26), we obtain the solution shown in (20) as required.

Corollary 17. Let $a(s)=\sqrt{1-4 h(s)}$. Then the solution to (18) is equal to

$$
t(s)= \begin{cases}1+c\left(\frac{a(s)+1}{a(s)-1}\right) e^{p_{1}(s)}, & \text { if } r(s)=r_{1}(s), \\ 1+c\left(\frac{a(s)-1}{a(s)+1}\right) e^{p_{2}(s)}, & \text { if } r(s)=r_{2}(s),\end{cases}
$$

where $p_{1}(s)$ and $p_{2}(s)$ are given by

$$
\begin{aligned}
p_{1}(s)= & \sum_{i=2}^{\lceil k / 2\rceil} \frac{2^{i-1}\left(\begin{array}{c}
2\lceil k / 2\rceil-i-1 \\
\lceil k / 2\rceil-i
\end{array}\right)}{(i-1)\left(\begin{array}{c}
k-1 \\
\lceil k / 2\rceil-1
\end{array}\right)} \\
& \times\left[\frac{(-1)^{i-1}}{(a(s)-1)^{i-1}}-\frac{1}{(a(s)+1)^{i-1}}\right], \\
p_{2}(s)= & \sum_{i=2}^{\lceil k / 2\rceil} \frac{2^{i-1}\left(\begin{array}{c}
2\lceil k / 2\rceil-i-1 \\
\lceil k / 2\rceil-i
\end{array}\right)}{(i-1)\left(\begin{array}{c}
k-1 \\
\lceil k / 2\rceil-1
\end{array}\right)} \\
& \times\left[\frac{(-1)^{i}}{(a(s)-1)^{i-1}}+\frac{1}{(a(s)+1)^{i-1}}\right] .
\end{aligned}
$$


Proof. We compute the integral in (20) for $r(s)=r_{1}(s)$. Let $l=\lceil k / 2\rceil, m=\left(\begin{array}{c}k-1 \\ \lceil k / 2\rceil-1\end{array}\right)$, and

$$
\begin{gathered}
a(s)=\left(1-4\left(\frac{s}{m}\right)^{1 / l}\right)^{1 / 2}, \\
r(s)=\frac{1}{2}+\frac{1}{2} a(s) .
\end{gathered}
$$

After some algebraic manipulations, we obtain the derivative $r^{\prime}(s)$ of $r(s)$ as follows:

$$
r^{\prime}(s)=\frac{1}{2} a^{\prime}(s) .
$$

By (29), we obtain the following expression for $s$ :

$$
s=\frac{\left(1-a(s)^{2}\right)^{l} m}{4^{l}} .
$$

Multiplying (31) by $1 / s$ and after some algebraic manipulations, we obtain

$$
\frac{1}{s} r^{\prime}(s)=\frac{4}{2 m} \frac{a^{\prime}(s)}{\left(1-a(s)^{2}\right)^{l}} .
$$

Based on (32) and (33), the integral in (20) becomes

$$
\int \frac{1}{s} r^{\prime}(s)=\frac{(-1)^{l} 4^{l}}{2 m} \int \frac{a^{\prime}(s)}{\left(a(s)^{2}-1\right)^{l}} d s .
$$

Setting $a(s)=w$ for convenience, we can form the rational parts expansion of the rational part of the integral in (34) as follows:

$$
\begin{aligned}
\frac{1}{\left(w^{2}-1\right)^{l}} & =\frac{1}{(w-1)^{l}(w+1)^{l}} \\
& =\sum_{i=1}^{l} \frac{A_{i}}{(w-1)^{i}}+\sum_{i=1}^{l} \frac{B_{i}}{(w+1)^{i}}
\end{aligned}
$$

with the coefficients given, by standard calculus techniques, by the following:

$$
\begin{aligned}
A_{i} & =\frac{1}{(l-i) !}\left[\frac{d^{(l-i)}}{d w^{(l-i)}}(w-1)^{l} \frac{1}{(w-1)^{l}(w+1)^{l}}\right]_{w=1} \\
& =\frac{1}{(l-i) !}\left[\frac{d^{(l-i)}}{d w^{(l-i)}} \frac{1}{(w+1)^{l}}\right]_{w=1} \\
B_{i} & =\frac{1}{(l-i) !}\left[\frac{d^{(l-i)}}{d w^{(l-i)}}(w+1)^{l} \frac{1}{(w-1)^{l}(w+1)^{l}}\right]_{w=-1} \\
& =\frac{1}{(l-i) !}\left[\frac{d^{(l-i)}}{d w^{(l-i)}} \frac{1}{(w-1)^{l}}\right]_{w=-1}
\end{aligned}
$$

for $i=1, \ldots, l$.
Since

$$
\frac{d^{(l-i)}}{d w^{(l-i)}} \frac{1}{(w \pm 1)^{l}}=(-1)^{l-i} \frac{1}{(w \pm 1)^{2 l-i}} \frac{(2 l-i-1) !}{(l-1) !},
$$

the coefficients $A_{i}$ and $B_{i}$ become

$$
\begin{aligned}
A_{i} & =\frac{1}{(l-i) !}(-1)^{l-i} \frac{1}{2^{2 l-i}} \frac{(2 l-i-1) !}{(l-1) !} \\
& =\left(\begin{array}{c}
2 l-i-1 \\
l-i
\end{array}\right)(-1)^{l-i} \frac{1}{2^{2 l-i}}, \\
B_{i} & =\frac{1}{(l-i) !}(-1)^{l-i} \frac{1}{(-2)^{2 l-i}} \frac{(2 l-i-1) !}{(l-1) !} \\
& =\left(\begin{array}{c}
2 l-i-1 \\
l-i
\end{array}\right) \frac{1}{(-1)^{l} 2^{2 l-i}} .
\end{aligned}
$$

Based on (34), (35), and (38), the integral in (20) becomes

$$
\begin{aligned}
\int \frac{1}{s} r^{\prime}(s) & \\
= & \frac{(-1)^{l} 4^{l}}{2 m} \int\left[\sum_{i=1}^{l} \frac{a^{\prime}(s) A_{i}}{(a(s)-1)^{i}}+\sum_{i=1}^{l} \frac{a^{\prime}(s) B_{i}}{(a(s)+1)^{i}}\right] \\
= & \frac{\left(\begin{array}{c}
2 l-2 \\
l-1
\end{array}\right)}{m} \ln \left(\frac{a(s)+1}{a(s)-1}\right)+\frac{(-1)^{l} 4^{l}}{2 m} \\
& \times \int\left[\sum_{i=2}^{l} \frac{a^{\prime}(s) A_{i}}{(a(s)-1)^{i}}+\sum_{i=2}^{l} \frac{a^{\prime}(s) B_{i}}{(a(s)+1)^{i}}\right] \\
= & \frac{\left(\begin{array}{c}
2 l-2 \\
l-1
\end{array}\right)}{m} \ln \left(\frac{a(s)+1}{a(s)-1}\right) \\
& +\sum_{i=2}^{l}\left[\frac{2^{i-1}\left(\begin{array}{c}
2 l-i-1 \\
l-i
\end{array}\right)}{(-1)^{i} m} \int \frac{a^{\prime}(s)}{(a(s)-1)^{i}}\right. \\
& \left.+\frac{2^{i-1}\left(\begin{array}{c}
2 l-i-1 \\
l-i
\end{array}\right)}{m} \int \frac{a^{\prime}(s)}{(a(s)+1)^{i}}\right] .
\end{aligned}
$$

Since $\left(\begin{array}{c}2 l-2 \\ l-1\end{array}\right)=m$, after some algebraic manipulations (39) is rewritten as follows:

$$
\int \frac{1}{s} r^{\prime}(s)=\ln \left(\frac{a(s)+1}{a(s)-1}\right)+p_{1}(s),
$$

where

$$
\begin{aligned}
p_{1}(s)=\sum_{i=2}^{l} \frac{2^{i-1}\left(\begin{array}{c}
2 l-i-1 \\
l-i
\end{array}\right)}{(i-1) m} \\
\quad \times\left[\frac{(-1)^{i-1}}{(a(s)-1)^{i-1}}-\frac{1}{(a(s)+1)^{i-1}}\right] .
\end{aligned}
$$

For $r(s)=r_{2}(s)$, the derivative $r^{\prime}(s)$ of $r(s)$ becomes

$$
r^{\prime}(s)=-\frac{1}{2} a^{\prime}(s) .
$$


Applying the previous proof method for $r^{\prime}(s)$ given by (42), the integral in (20) becomes

$$
\int \frac{1}{s} r^{\prime}(s) d s=\ln \left(\frac{a(s)-1}{a(s)+1}\right)+p_{4}(s),
$$

where

$$
\begin{aligned}
p_{2}(s)=\sum_{i=2}^{l} \frac{2^{i-1}\left(\begin{array}{c}
2 l-i-1 \\
l-i
\end{array}\right)}{(i-1) m} \\
\quad \times\left[\frac{(-1)^{i}}{(a(s)-1)^{i-1}}+\frac{1}{(a(s)+1)^{i-1}}\right] .
\end{aligned}
$$

Thus, from (20), we obtain the following:

$$
t(s)= \begin{cases}1+c\left(\frac{a(s)+1}{a(s)-1}\right) e^{p_{1}(s)}, & \text { if } r(s)=r_{1}(s), \\ 1+c\left(\frac{a(s)-1}{a(s)+1}\right) e^{p_{2}(s)}, & \text { if } r(s)=r_{2}(s),\end{cases}
$$

completing the proof of the theorem.

We now need to invert the function $t(s)$ or, equivalently, to solve (27), with unknown $s$. By setting $z=1+a(s)$, (27) for $r(s)=r_{2}(s)$ after some algebraic manipulations becomes

$$
z=\frac{c(z-2)}{t(s)-1} e^{\mathrm{Q}(z)}
$$

where $Q(z)$ is the rational function of $z$ given by

$$
\begin{aligned}
Q(z)=\sum_{i=2}^{l} \frac{2^{i-1}\left(\begin{array}{c}
2 l-i-1 \\
l-i
\end{array}\right)}{(i-1) m} \\
\times \frac{(-1)^{i} z^{i-1}+(z-2)^{i-1}}{z^{i-1}(z-2)^{i-1}} .
\end{aligned}
$$

With regard to the constant $c$, its value may be obtained from $x(0)$ as follows. Since $y(t)=x(t) /(1-t)$, then $y(0)=x(0)$. Setting $t=0$ in $g(y(t))=s$, we obtain the value for $s$. Thus, using this value of $s$, we have established the relation $t(s)=0$, and we can compute $a(s)$ and $z$. Now we can solve (46) for $c$.

Setting

$$
f(z)=\frac{\ln (c(2-z) /(1-t(s)))}{e^{z}}+\frac{Q(z)}{e^{z}},
$$

we rewrite (46) as follows:

$$
z=e^{f(z) e^{z}} .
$$

Based on Definitions 12, 13, and 14 for $j=1$, we can rewrite (49) as follows:

$$
\begin{aligned}
z & =e^{f(z) e^{z}} \\
& \Longrightarrow z e^{-f(z) e^{z}}=1 \\
& \Longrightarrow G(-f(z) ; z)=1 \\
& \Longrightarrow z=\mathrm{HW}(-f(z) ; 1) .
\end{aligned}
$$

Let $l=\lceil k / 2\rceil$ and $m=\left(\begin{array}{c}k-1 \\ \lceil k / 2\rceil-1\end{array}\right)$. Since $z=1+a(s)$ by substituting $a(s)$ and after some algebraic manipulations, we obtain the following formula for $s$ :

$$
\begin{aligned}
s & =\frac{m z^{l}(z-2)^{l}}{4^{l}} \\
& =\frac{m \mathrm{HW}(-f(z) ; 1)^{l}(\mathrm{HW}(-f(z) ; 1)-2)^{l}}{4^{l}} .
\end{aligned}
$$

Equation (51) gives values of $s$ for specific values of $t$ through Hyper-Lambert functions. Specifically, for a certain value of $t$, the function $f(z)$ takes a particular form, and through (51), we obtain a value for $s$. Thus, we have found $t^{-1}(s)$. In [6], (51) has been derived for $k=3$.

Based on Theorem 16, Lemma 15, (50), and that $r(s)=$ $z / 2$, a solution of the differential equation (13) can be obtained by

$$
y(t(s))=r(s)=\frac{\operatorname{HW}(-f(z) ; 1)}{2} .
$$

Equivalently, we can obtain the solution by

$$
y(t(s))=r\left(\frac{m \mathrm{HW}(-f(z) ; 1)^{l}(\mathrm{HW}(-f(z) ; 1)-2)^{l}}{4^{l}}\right)
$$

with $r(s)=r_{2}(s)$.

We will now demonstrate the previous methodology for $k=3$. Since $l=\lceil k / 2\rceil=2$ and $m=\left(\begin{array}{c}k-1 \\ \lceil k / 2\rceil-1\end{array}\right)=2,(21)$ becomes

$$
\begin{aligned}
g(y(t)) & =2 y(t)^{2}(1-y(t))^{2} \\
& =2 y(t)^{4}-4 y(t)^{3}+2 y(t)^{2} .
\end{aligned}
$$

From Theorem 16, the real positive solutions $r_{1}(s)$ and $r_{2}(s)$ of $g(y(t))=s$ for $s \leq(1 / 8)$ can be written as follows:

$$
r_{1}(s)=\frac{1+\sqrt{1-2 \sqrt{2 s}}}{2}, \quad r_{2}(s)=\frac{1-\sqrt{1-2 \sqrt{2 s}}}{2}
$$

Moreover, the rational function $Q(z)$ of $z$ involved in (46) becomes, after some algebraic manipulations, as follows:

$$
Q(z)=\frac{2(z-1)}{z(z-2)}
$$

Thus, the function $f(z)$ defined by (48) becomes

$$
f(z)=\frac{\ln (c(2-z) /(1-t(s)))}{e^{z}}+\frac{2(z-1)}{z(z-2) e^{z}} .
$$


Finally, the formula for $s$ defined by (51) and the solution of the differential equation (54) defined by (53) can be written as follows:

$$
\begin{aligned}
s & =\frac{z^{2}(z-2)^{2}}{8} \\
& =\frac{\operatorname{HW}(-f(z) ; 1)^{2}(\mathrm{HW}(-f(z) ; 1)-2)^{2}}{8} . \\
y(t(s)) & =r\left(\frac{\operatorname{HW}(-f(z) ; 1)^{2}(\mathrm{HW}(-f(z) ; 1)-2)^{2}}{8}\right),
\end{aligned}
$$

where $r(\cdot)=r_{2}(s)$.

\section{Nonclosed-Form Solvability Results for Even Values of $k$}

In this section, we consider the case where the parameter $k$ is an even integer greater than 2 , that is, $k=2 m$, with $m \geq 2$. This case is of distinct interest because it does not seem to be amenable to the methodology we have followed for $k$ odd. In Section 3 where we considered odd values of $k$, a key step in linking the solution of the differential equation with the class of Hyper-Lambert functions was to find the closed-form solution $y(t)$, given $s$, of the polynomial equation $g(y(t))=$ $s$, with $g(y(t))$ given by (21). This closed-form solution was needed in Lemma 15 and Theorem 16. When we attempted to apply the same methodology for even values of $k \geq 4$, we were not able to find a closed-form solution of the equation $g(y(t))=s$, even for fixed value of $k$ (e.g., $k=4)$. Thus, we focused our attention on investigating the possibility that the equation does not have a closed-form solution. As it turned out, this was indeed the case, at least for values of $k$ such that $k+1$ is prime and certain ranges of $s$. We believe that this result may be of independent interest.

Before proving it, we will state three fundamental theorems of Algebra.

Theorem 18 (Eisenstein's irreducibility criterion). Let $q$ be $a$ prime number, and let $f(x)=a_{n} x^{n}+\cdots+a_{1} x^{1}+a_{0}$ be a polynomial of degree $n$ with integer coefficients satisfying the following properties:

(i) $q$ does not divide $a_{n}$;

(ii) $q$ divides $a_{0}, a_{1}, \ldots, a_{n-1}$;

(iii) $q^{2}$ does not divide $a_{0}$.

Then the polynomial $f$ is irreducible over the field of $\mathbb{Q}$ of rational numbers.

Theorem 19. Let $K$ be a subfield of $\mathbb{R}$, and let $f \in K[X]$ be an irreducible polynomial of prime degree $n>2$. If the Galois group of $f$ over $K$ is solvable, $f$ has either exactly one root in $\mathbb{R}$ or all its roots in $\mathbb{R}$.

Theorem 20. Let $f$ be a polynomial with coefficients in a field $K$ of characteristic zero. Suppose that $f$ is solvable by radicals. Then the Galois group of $\Gamma_{K}(f)$ of $f$ is a solvable group.
We now state and prove the main result, concerning the unsolvability, in closed form, of the polynomial equation $g(y(t))=s$.

Theorem 21. The equation $g(y(t))=s$ is not solvable by radicals over $\mathbb{Q}$ for $k=2 m$ with $m \geq 2, n=k+1$ a prime, and $s=l / b$ a rational number, given in its lower terms (i.e., $\operatorname{gcd}(l, b)=1)$, such that $s \in\left(0, g\left(w_{3}\right)\right), l b \neq 0$, and $b$ is a square free integer.

Proof. Let $s=l / b \in \mathbb{Q}$, with $l b \neq 0$. Then, our target polynomial is $p(y(t))=g(y(t))-(l / b)$. We will, first, show that $p(y(t))$ is irreducible over the set of rational numbers $\mathbb{Q}$ using Theorem 18. We will work, in the following, with the polynomial $b p(y(t))$ in order to have a polynomial with integer coefficients, as required by the statement of Theorem 18. The degree of this polynomial according to Theorem 8 is $n=k+1$, which has been assumed to be a prime.

We will show, however, that Theorem 18 is not, directly, applicable to $b p(y(t))$ since the first two conditions of the theorem are not met. The coefficients $a_{i}, i=0, \ldots, n$ in Theorem 18 are as follows:

$$
\begin{gathered}
a_{i}=b\left(\begin{array}{c}
2 m-1 \\
m-1
\end{array}\right)\left(\begin{array}{c}
m \\
i-(m+1)
\end{array}\right)(-1)^{i-(m+1)}, \\
i=m+1, \ldots, \quad n=2 m+1, \\
a_{i}=0, \quad i=1, \ldots, m, \\
a_{0}=-l .
\end{gathered}
$$

Since $\left|a_{n}\right|=a_{m+1}=b\left(\begin{array}{c}2 m-1 \\ m-1\end{array}\right)$, the first two conditions of Theorem 18 do not hold. Since 0 is not a root of $p(y(t))$, we can set $y(t) \rightarrow 1 / x$, in order to consider the polynomial $f(x)=b p(1 / x)$, instead of $b p(y(t))$. Then each nonzero root $x_{0}$ of the polynomial $f(x)$ corresponds to a nonzero root $y_{0}$ of $p(y(t))$ and vice versa by the correspondence $y_{0} \leftrightarrow 1 / x_{0}$. Consequently, $p(y(t))$ is solved by radicals if and only if $f(x)$ is solved by radicals.

Thus, from now on we will consider the polynomial $f(x)=b p(1 / x)$. In order to have the inverse of $1 / x$, we will use the polynomial $h(x)=x^{2 m+1} f(x)$. We will apply Theorem 18 to this polynomial because if it applies to it, then $f(x)$ will be irreducible, as well as, in turn, $p(y(t))$.

After some algebraic manipulations, the polynomial $h(x)=x^{2 m+1} f(x)$ can be written as follows:

$$
h(x)=b\left(\begin{array}{c}
2 m-1 \\
m-1
\end{array}\right) \sum_{i=0}^{m}\left(\begin{array}{c}
m \\
i
\end{array}\right) x^{m-i}(-1)^{i}-l x^{2 m+1}
$$

Now the coefficients $a_{i}, i=0, \ldots, n=2 m+1$ of the polynomial $h(x)$ in $(60)$ are as follows:

$$
\begin{gathered}
a_{2 m+1}=-l, \\
a_{j}=0, \quad j=m+1, \ldots, 2 m, \\
a_{j}=b\left(\begin{array}{c}
2 m-1 \\
m-1
\end{array}\right)\left(\begin{array}{c}
m \\
m-j
\end{array}\right)(-1)^{m-j}, \quad j=0, \ldots, m .
\end{gathered}
$$


In this way, we have decoupled the coefficient $a_{n}=a_{2 m+1}$ from all the rest so as to be feasible to satisfy the first two conditions of Theorem 18 . Since, by assumption, $\operatorname{gcd}(l, b)=1$, in order to satisfy the first two conditions, it suffices to choose as $q$ any prime divisor of $b$. Since $b$ is, by assumption, a square free integer, then the third condition is satisfied too. Thus, Theorem 18 applies to $h(x)$ in $(60)$ and, thus, to $p(y(t))$. Therefore, $p(y(t))$ is irreducible over $\mathbb{Q}$.

We will, now, prove that the number of real roots of $h(x)$ is neither equal to 1 nor its degree, which is $n=k+1$. From Theorem 10, since we have assumed that $s \in\left(0, g\left(w_{3}\right)\right)$, it follows that $g(y(t))$ has at least two roots and, thus, $h(x)$ has also at least two roots because of the relationship between their roots. We will prove that not all of its roots are real. Let us assume, towards a contradiction, that $h(x)$ has $2 m+1$ real roots. We can differentiate $h(x) m$ times to obtain its $m$ th order derivative, which is equal to $l((2 m+1) ! / m !) x^{m+1}+$ $m ! b\left(\begin{array}{c}2 m-1 \\ m-1\end{array}\right)$. If $h(x)$ had $2 m+1$ real roots, then its $m$ th order derivative would have, at least, $2 m+1-m=m+1 \geq 3$ (since $m \geq 2$, by assumption) real roots.

The roots of $l((2 m+1) ! / m !) x^{m+1}+m ! b\left(\begin{array}{c}2 m-1 \\ m-1\end{array}\right)$ are the $(m+1)$ th roots of $-B / A$, with $A=l((2 m+1) ! / m !)$ and $B=m ! b\left(\begin{array}{c}2 m-1 \\ m-1\end{array}\right)$. These roots have the form $|-B / A|^{1 /(m+1)}$ $(\cos ( \pm \pi+2 d \pi) /(m+1)+i \sin ( \pm \pi+2 d \pi) /(m+1))$ with $d=0, \ldots, m( \pm \pi$ is $\pi$ if $-B / A$ is positive and $-\pi$ if $-B / A$ is negative).

A root is real if and only if the imaginary part is 0 ; that is, $( \pm \pi+2 d \pi) /(m+1)=\pi((2 d \pm 1) /(m+1))$ is an integer multiple $\lambda$ of $\pi$. Since $d \leq m$, we only need to consider $\lambda=1$. Solving $(2 d \pm 1) /(m+1)=1$, we obtain $d=(m+1 \mp 1) / 2$. It is easy to see that this gives us at most two values for $d$ : (i) two values for even $m: m / 2$ (for the "-" sign in $(2 d \pm 1) /(m+1)$ ) and $(m+2) / 2$ (for the "+" sign in $(2 d \pm 1) /(m+1))$ and (ii) no such value for odd $m$. Thus, the $m$ th order derivative of $h(x)$ has at most 2 real roots for even $m$ or no real roots for odd $m$, while according to the assumption that $h(x)$ has $2 m+1$ real roots, it should have at least $m+1 \geq 3$ real roots, which is a contradiction.

Therefore, either there are no real roots of $h(x)$, or the number of real roots of $h(x)$ is more than 1 but less than its degree $n=2 m+1$. Thus, from Theorems 19 and 20, it follows that $h(x)$ is not solvable by radicals over $\mathbb{Q}$. Therefore, $p(y(t))$ is not solvable by radicals over $\mathbb{Q}$ too.

\section{Conclusions and Future Work}

In this paper we studied the properties of the solution of the differential equation

$$
(1-t) \frac{d y(t)}{d t}=\left(\begin{array}{c}
k-1 \\
\left\lceil\frac{k}{2}\right\rceil-1
\end{array}\right) y(t)^{k-\lceil k / 2\rceil+1}(1-y(t))^{\lceil k / 2\rceil}
$$

This differential equation arose out of the theoretical analysis of a security-related bit-agreement protocol (see [1]) and does not appear to have been documented elsewhere. It can be seen as a subclass of a natural generalization of the well-known class of Abel differential equations of the first kind.
In this paper we showed that, for odd $k$, the solution of this equation can be expressed in closed form through the roots of the polynomial on its right-hand side. Moreover, the resulting expression can be transformed into a closedform expression involving the generalized Hyper-Lambert functions that were proposed in [3] as a generalization of the well-known Lambert $W$ function.

For even values of $k$ it was not possible to follow the same approach since, as we showed, no closed-form expression exists for the roots of the polynomial, at least when $k+1$ is prime. One possible direction for future research is to extend the proof to cover all value of $k$ or show that closed-form solutions exist for nonprime $k$.

Another, more interesting direction would be to see whether the more general class given in (2) can be attacked using the strategy proposed in this paper, that is, based on deriving closed-form solutions of the polynomial on the right-hand side and linking them with the solution of the differential equation, at least for certain polynomials $f_{i}$. There seems to be very scarce research work on the properties of (2), and any result towards the determination of its solution or, at least, the properties of its properties would be of high importance.

\section{References}

[1] E. Makri and Y. C. Stamatiou, "An interactive, similarity increasing algorithm for random strings with applications to key agreement in ad hoc networks," Studies in Applied Mathematics, vol. 121, no. 2, pp. 141-155, 2008.

[2] P. E. Nastou, Y. C. Stamatiou, and A. Tsiakalos, "Solving a class of ODEs arising in the analysis of a computer security process using generalized Hyper-Lambert Functions," International Journal of Applied Mathematics and Computation, vol. 4, no. 3, pp. 285-294, 2012.

[3] I. N. Galidakis, "On some applications of the generalized hyperLambert functions," Complex Variables and Elliptic Equations, vol. 52, no. 12, pp. 1101-1119, 2007.

[4] R. M. Corless, G. H. Gonnet, D. E. G. Hare, D. J. Jeffrey, and D. E. Knuth, "On the lambert $W$ function," Advances in Computational Mathematics, vol. 5, no. 4, pp. 329-359, 1996.

[5] G. M. Murphy, Ordinary Differential Equations and Their Solutions, D. Van Nostrand, 1960.

[6] P. E. Nastou, Y. C. Stamatiou, and A. Tsiakalos, “The solution of dierential equation describing the evolution of a key agreement protocol," in EUROSIAM, Montreux, Switzerland, 2011. 


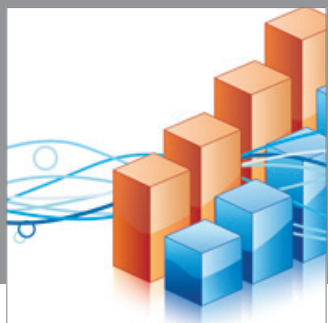

Advances in

Operations Research

mansans

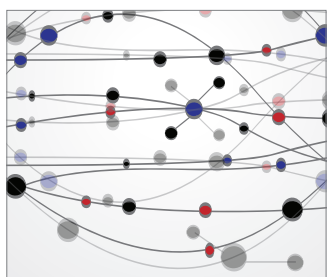

The Scientific World Journal
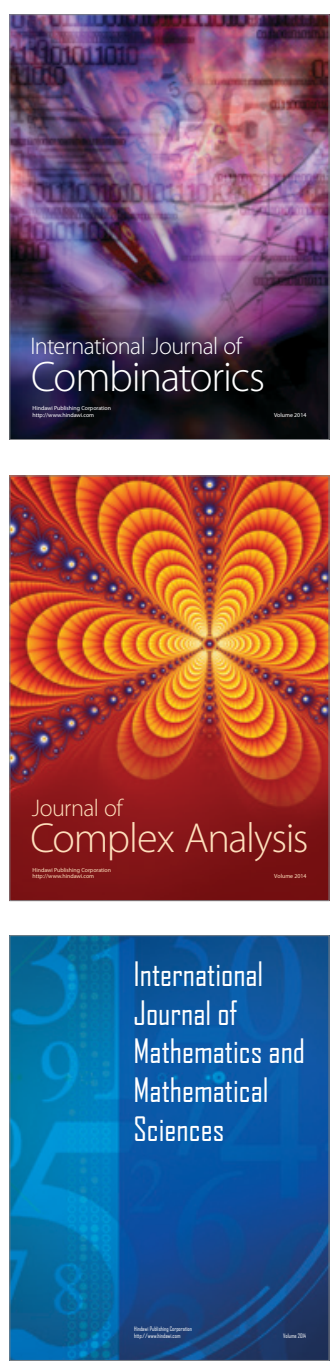
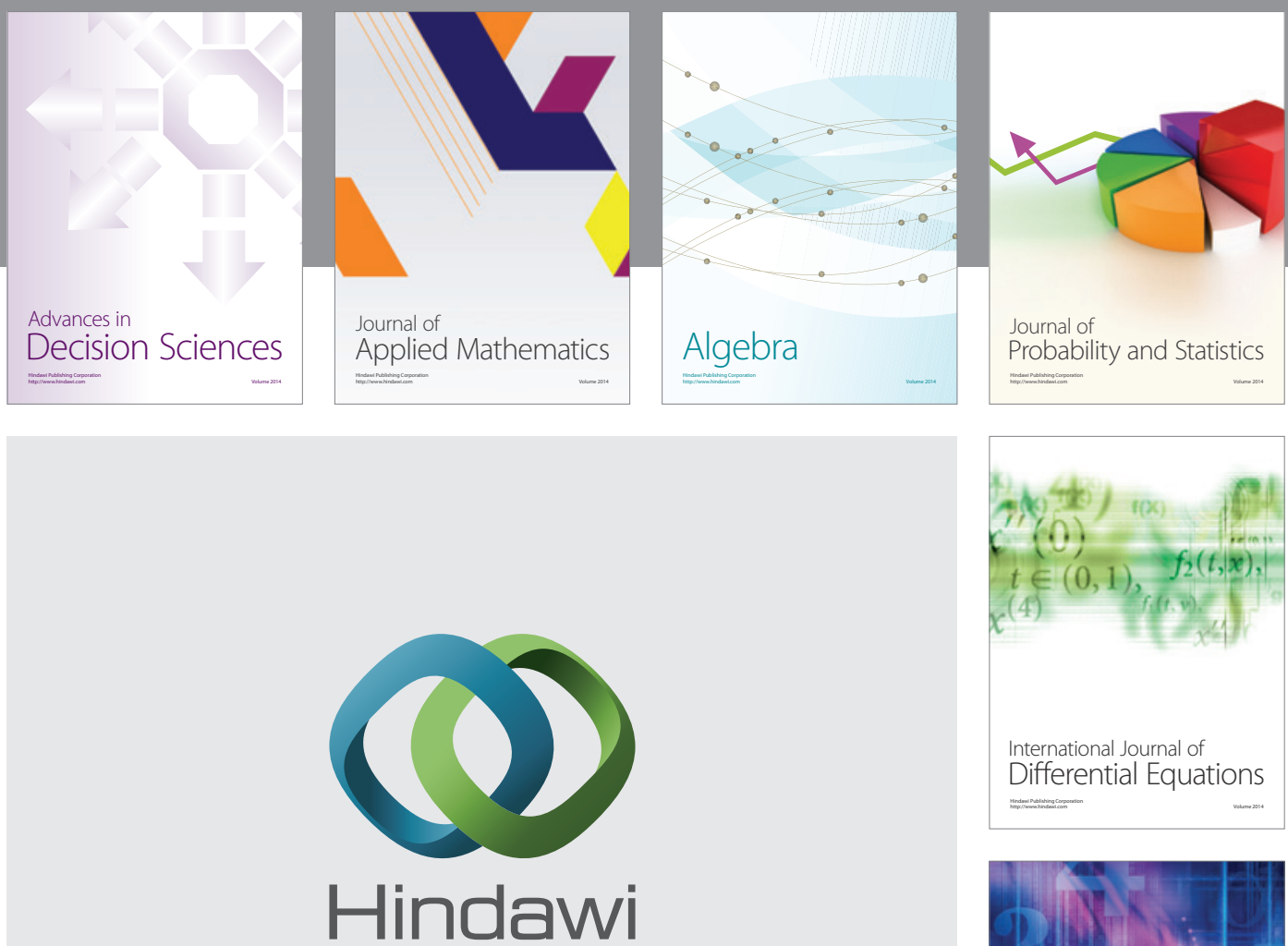

Submit your manuscripts at http://www.hindawi.com
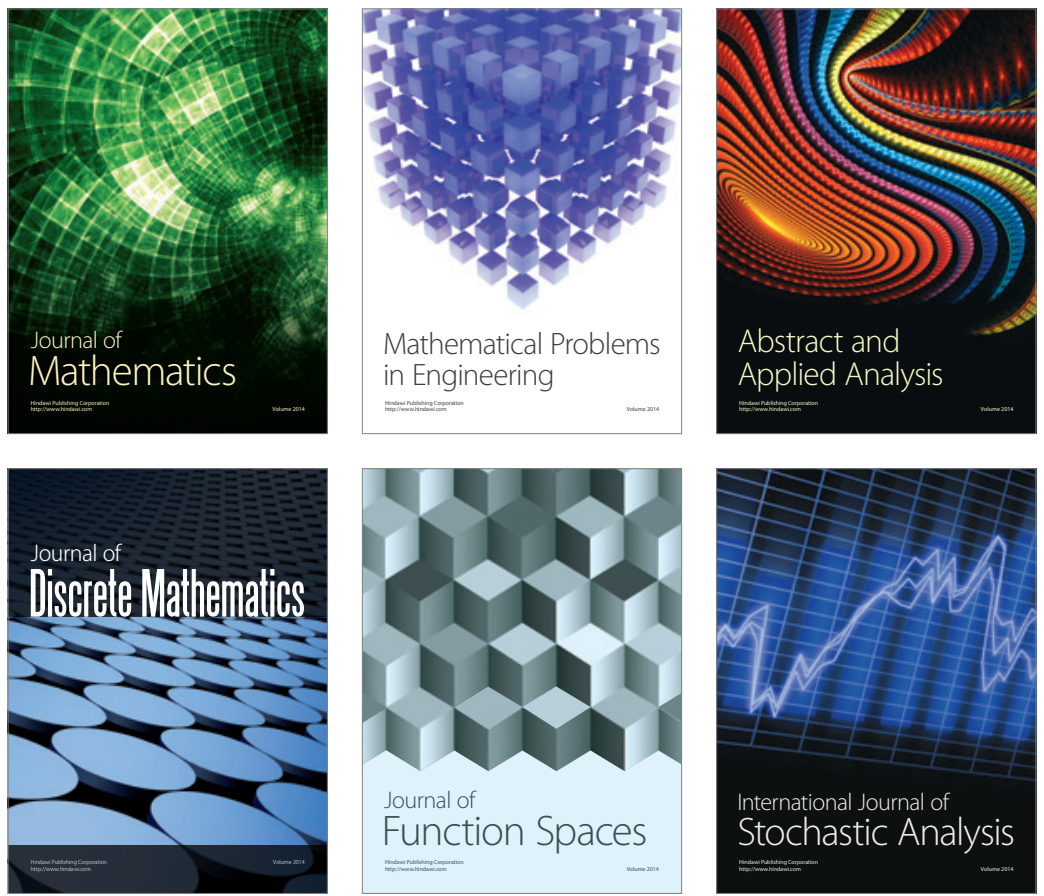

Journal of

Function Spaces

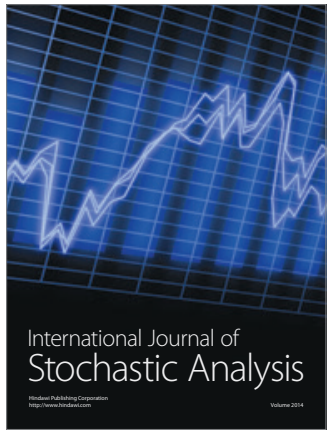

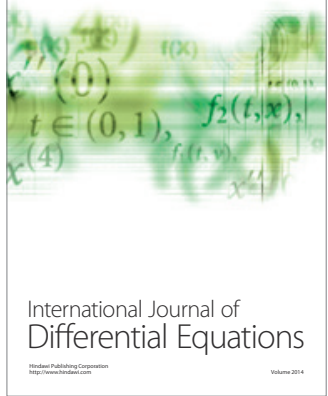
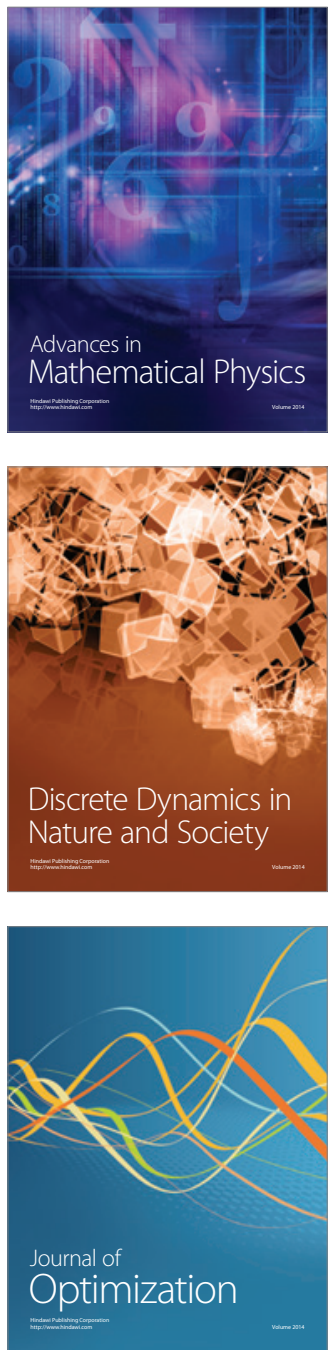